\title{
Estimating inter-area dominant oscillation mode in bulk power grid using multi-channel continuous wavelet transform
}

\author{
Tao JIANG ${ }^{1}$, Linquan BAI ${ }^{2}$, Guoqing LI $^{1}$, Hongjie $\mathrm{JIA}^{\mathbf{3}}$, \\ Qinran $\mathrm{HU}^{4}$, Haoyu YUAN ${ }^{2}$
}

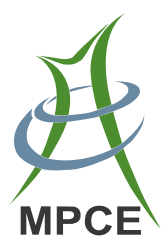

\begin{abstract}
This paper proposes a novel continuous wavelet transform (CWT) based approach to holistically estimate the dominant oscillation using measurement data from multiple channels. CWT has been demonstrated to be effective in estimating power system oscillation modes. Using singular value decomposition (SVD) technique, the original huge phasor measurement unit (PMU) datasets are compressed to finite useful measurement data which contain critical dominant oscillation information. Further, CWT is performed on the constructed measurement signals
\end{abstract}

CrossCheck date: 4 May 2016

Received: 28 September 2015/Accepted: 4 May 2016/Published online: 30 June 2016

(C) The Author(s) 2016. This article is published with open access at Springerlink.com

$\triangle$ Tao JIANG

tjiang@nedu.edu.cn

Linquan BAI

lbai3@tuk.edu

Guoqing LI

lgq@nedu.edu.cn

Hongjie JIA

hjjia@tju.edu.cn

Qinran HU

qh@ieee.org

Haoyu YUAN

hyuan2@vols.utk.edu

1 Department of Electrical Engineering, Northeast Dianli University, Jilin 132012, China

2 Department of Electrical Engineering and Computer Science, University of Tennessee, Knoxville, TN 37996, USA

3 Key Laboratory of Smart Grid of Ministry of Education, Tianjin University, Tianjin 300072, China

4 School of Engineering and Applied Sciences, Harvard University, Cambridge, MA 02138, USA to form wavelet coefficient matrix (WCM) at the same dilation. Then, SVD is employed to decompose the WCMs to obtain the maximum singular value and its right eigenvector. A singular value vector with the entire dilation is constructed through the maximum singular values. The right eigenvector corresponding to the maximum singular value in the singular-value vector is adopted as the input of CWT to estimate the dominant modes. Finally, the proposed approach is evaluated using the simulation data from China Southern Power Grid (CSG) as well as the actual field-measurement data retrieved from the PMUs of CSG. The simulation results demonstrate that the proposed approach performs well to holistically estimate the dominant oscillation modes in bulk power systems.

Keywords Continuous wavelet transform (CWT), Oscillation mode, Phasor measurement unit (PMU), Singular value decomposition (SVD)

\section{Introduction}

Small signal stability is an ability of power system to maintain its synchronism when subjected to small disturbances [1-4]. As a useful tool to analyze nonlinear dynamic systems, modal analysis is usually adopted to study power system small-signal stability. At a given operating point, nonlinear differential algebraic equations (DAEs) of the system can be linearized by modal analysis. Then, the eigenvalues of the state matrix of the linearized system model can be calculated to estimate the oscillation mode and its shape [5-7]. Hence, the modelbased method enables system operators to assess the power system dynamic features at certain operation points. However, since the power system operating point 
is changing all the time as a result of load, generation variations and system contingencies, it is challenging to maintain a detailed power system model with accurate parameters. Moreover, the estimated parameters of the model are only effective for a bounded neighborhood of certain operating point, and the computational burden will dramatically increase with the growth of model size. Due to the aforementioned limitations, the implementations of such model-based approaches are limited to off-line small signal stability analysis.

Motivated by the fast development and wide applications of synchronous phasor measurement units (PMUs) in power systems, several measurement-based methods have been proposed in literature and implemented in practical power systems [8-16]. Such approaches can directly estimate the dominant oscillation modes of power systems from PMU data. Generally, they can be classified into two categories: ringdown-based approaches and ambient-based approaches $[9,10]$.

On one hand, the ringdown-based approaches estimate dominant oscillation modes from the system response incurred by sudden disturbances including line tripping, generator outage, bus fault, etc. According to existing literature, several ringdown-based approaches have been discussed. Prony was firstly conducted by [11] to estimate the WECC dominant modes. This was a pioneering article that initiated the application of identification methods to study power system oscillation, but it is unable to separate the dominate modes from the trivial modes to reduce the number of false alarms. To address this challenge in mode estimation, a stepwise-regression based Prony method was further developed in [10] to automatically identify dominant electro-mechanical modes. However, this method may increase the computational burden and its performance becomes much worse with high-level noises in the measurement data. To suppress the noises in the mode estimation from ringdown data, a mode matching method based on subspace methods was developed in [8] to analyze the small signal stability of China Southern Power Grid (CSG) using PMU data, but the performance of this method depended on the operational experience. It cannot track the dominant modes when the operating point of the system changes. To solve this problem, continuous wavelet transform (CWT) was proposed in [12] to exploit the relationship between low-frequency oscillation features, and then the Morlet-based CWT of ringdown data was proposed to detect modal parameter changes and several guidelines were designed for selecting the center frequency, bandwidth parameters, scaling factor and the translation factor of CWT.

On the other hand, the ambient-based approaches identify the dominant oscillation modes from the ambient data excited by small random load or generation fluctuations in power systems. To estimate the oscillation modes from ambient data, covariance-driven stochastic subspace identification (COV-SSI) with reference channel was applied in [13], to automatically detect the real modes. The concept of point density based on stabilization diagram was also defined, but such method was not capable of automatically identifying the dominant modes and the model order determination remains an issue for the COV-SSI. To surmount the shortcoming of the proposed method in [13], CWT is employed in [14] to estimate the oscillation frequency, and a combination of CWT and random decrement technique (RDT) is used to estimate the damping ratio. The proposed method was further applied in Nordic power system. Reference [15] proposed orthogonal CWT to detect the dominant modes from ambient data.

Among the aforementioned methods, CWT is an effective technique for analyzing nonstationary signals, and it can capture the dynamic features of power systems in both time and frequency domains $[12,14]$. However, CWT is a typical single-channel identification algorithm that is unable to simultaneously process multi-channel measurement data [16]. To estimate the oscillation mode from multi-channel measurement data, CWT has to be implemented on the measurement data of each channel one by one, which may aggravate the computational burden. Moreover, due to the measurement noises and calculation errors, the estimated oscillation frequency and damping from different channels may not be consistent. This inconsistency may prevent system operators from taking timely actions to maintain system dynamic stability, which may further lead to an outage.

Motivated by these existing issues, this paper proposes a multi-channel CWT-based (MCWT) mode estimation approach. In order to improve the computational efficiency of CWT in processing multi-channel measurement data, a data compression technique is developed to remove the redundancies and retain the dominant components. The data compression technique consists of two parts: the first part based on SVD is responsible for decomposing the covariance matrix generated by the multi-channel measurement signals; the second part is to construct measurement signal using the results of SVD with a proposed model order determination method. For the constructed measurement signal, CWT is applied to form wavelet coefficient matrix (WCM) at the same dilation. With the WCMs, a singular-value vector is constructed using the maximum singular value of each WCM obtained through SVD. In this singular value vector, the maximum component is considered as the dominant oscillation mode indicator to select the right-singular vector. Using the selected right vectors, the dominant modes can be estimated holistically. 
The highlighted contributions of this paper can be summarized as follows.

1) A framework of multi-channel CWT approach for estimating inter-area dominant oscillation modes from the multi-channel measurements is developed.

2) A general model order determination strategy for the measurement data reconstruction is proposed.

3) An architecture of measurement data compression and reconstruction for the proposed MCWT mode estimation is characterized using the SVD.

\section{Continuous wavelet transform}

CWT is a time-frequency transform method which decomposes a signal over the wavelets using specific dilations and translations. Unlike Fourier transform (FT) $[17,18]$, CWT is able to construct a time-frequency representation of a signal [14]. Hence, it has been widely used in various fields of power systems studies including load forecasting, fault detection, transformer differential protection, broken rotor bar diagnosis, etc.

In this paper, CWT is employed to estimate power system dominant oscillation modes based on PMU measurement data. The procedure of mode estimation using CWT is briefly described as follows:

For a continuous time signal $x(t)$, the CWT of $x(t)$ can be expressed as $[12,14]$

$$
\begin{aligned}
W(\tau, s) & =\frac{1}{\sqrt{\tau}} \int_{-\infty}^{+\infty} x(t) \psi^{*}\left(\frac{t-s}{\tau}\right) \mathrm{d} t \\
& =\int_{-\infty}^{+\infty} x(t) \psi_{\tau, s}^{*}(t) \mathrm{d} t=\left\langle x(t), \psi_{\tau, s}(t)\right\rangle
\end{aligned}
$$

where $W(\tau, s)$ is the wavelet coefficient of $x(t) ; \tau$ is the dilation; $s$ is the translation; $\Psi_{\tau, s}$ is the mother wavelet function.

Considering the case of $x(t)$ with one oscillation mode, $x(t)$ can be represented as

$x(t)=A(t) \cos (\omega t+\delta)$

where $A(t)$ is the magnitude of $x(t) ; \omega$ is the angular frequency; $\delta$ is the phase angle.

Substituting (2) into (1) and considering the mother wavelet function $\Psi_{\tau, s}$ as the complex Morlet wavelet, the wavelet coefficient of $x(t)$ in (2) is expressed as

$$
\begin{aligned}
W(\tau, s) & =\frac{1}{\sqrt{\tau}} \int_{-\infty}^{+\infty} x(t) \psi_{\tau, s}^{*}(t) \mathrm{d} t \\
& =\frac{\sqrt{\tau}}{2} A(s) \hat{\psi}_{\tau, s}^{*}(\tau \omega) \mathrm{e}^{\mathrm{j} \omega s+\delta}
\end{aligned}
$$

Similarly, if $x(t)$ contains multiple oscillation modes, then $x(t)$ can be further expressed as $x(t)=\sum_{i=1}^{m} A_{i} \mathrm{e}^{-\varsigma_{i} \omega_{n i} t} \cos \left(\omega_{d i} t+\delta_{0 i}\right)$

According to (3), the wavelet coefficient of $x(t)$ can be reformulated as

$W(\tau, t)=\frac{\sqrt{\tau}}{2} \sum_{i=1}^{m} A_{i} \mathrm{e}^{-\varsigma_{i} \omega_{n i} t} \hat{\psi}_{\tau, t}^{*}\left(\tau \omega_{d i}\right) \mathrm{e}^{\mathrm{j}\left(\omega_{d i} t+\delta_{0 i}\right)}$

where $A_{i}$ and $\delta_{0 i}$ are the magnitude and initial phase angle of $i^{\text {th }}$ component of $x(t)$ with respect to the $i^{\text {th }}$ oscillation mode; $\varsigma_{i}, \omega_{n i}, \omega_{d i}$ are the damping, undamped angular frequency and damped angular frequency of $i^{\text {th }}$ oscillation mode, respectively.

Considering the linear combination property of CWT, the wavelet coefficient of the $i^{\text {th }}$ component in $x(t)$, which can be extracted from (5), is defined as

$W\left(\tau_{i}, t\right)=\frac{\sqrt{\tau_{i}}}{2} A_{i} \mathrm{e}^{-\varsigma_{i} \omega_{n i} t} \hat{\psi}_{\tau, t}^{*}\left(\tau \omega_{d i}\right) \mathrm{e}^{\mathrm{j}\left(\omega_{d i} t+\delta_{0 i}\right)}$

For (6), the modulus of $W\left(\tau_{i}, t\right)$ is

$\left|W\left(\tau_{i}, t\right)\right|=\frac{\sqrt{\tau_{i}}}{2} A_{i} \mathrm{e}^{-\varsigma_{i} \omega_{n i} t}\left|\hat{\psi}_{\tau, t}^{*}\left(\tau \omega_{d i}\right)\right|$

Applying the logarithmic and derivation to (7), we have

$$
\begin{aligned}
\ln \left|W\left(\tau_{i}, s\right)\right| & =\ln \left(\frac{\sqrt{\tau_{i}}}{2} A_{i} \mathrm{e}^{-\varsigma_{i} \omega_{n i} t}\left|\hat{\psi}_{\tau, t}^{*}\left(\tau \omega_{d i}\right)\right|\right) \\
& =-\varsigma_{i} \omega_{n i} t+\ln \left(\frac{\sqrt{\tau_{i}}}{2} A_{i}\left|\hat{\psi}_{\tau, t}^{*}\left(\tau \omega_{d i}\right)\right|\right)
\end{aligned}
$$

$\frac{\mathrm{d}\left(\ln \left|W\left(\tau_{i}, t\right)\right|\right)}{\mathrm{d} t}=-\varsigma_{i} \omega_{n i}$

The phase angle can be derived from (6), expressed as $\operatorname{angle}\left(W\left(\tau_{i}, t\right)\right)=\omega_{d i} t+\delta_{0 i}$

Calculating the derivation of (10) with respect to $t$, we can further achieve

$\frac{\mathrm{d}\left(\operatorname{angle}\left(W\left(\tau_{i}, t\right)\right)\right)}{\mathrm{d} t}=\omega_{d i}=\omega_{n i} \sqrt{1-\varsigma_{i}^{2}}$

According to (9) and (11), the un-damped angular frequency of the $i^{\text {th }}$ oscillation mode can be calculated as

$\omega_{n i}=\sqrt{\left(\frac{\mathrm{d}\left(\ln \left|W\left(\tau_{i}, t\right)\right|\right)}{\mathrm{d} t}\right)^{2}+\left(\frac{\mathrm{d}\left(\operatorname{angle}\left(W\left(\tau_{i}, t\right)\right)\right)}{\mathrm{d} t}\right)^{2}}$

From (8) and (12), the frequency $f_{i}$ and damping $\varsigma_{i}$ of the $i^{\text {th }}$ oscillation mode contained in $x(t)$ are

$\left\{\begin{array}{l}f_{i}=\frac{\omega_{n i}}{2 \pi} \\ \varsigma_{i}=-\frac{\frac{\mathrm{d}\left(\ln \left|W\left(\tau_{i}, t\right)\right|\right)}{\mathrm{d} t}}{\omega_{n i}}\end{array}\right.$ 
Hence, the procedure of power system mode estimation through CWT can be summarized as follows: For a measurement data $x(t)$, CWT is applied to the measurement data to obtain the wavelet coefficients of dominant modes. Then, the modes based on the obtained wavelet coefficients can be detected through (7)-(13).

\section{Proposed approach}

The properties of CWT-based identification algorithm are as follows. This algorithm is only applicable to the mode identification for single-channel signal. When implemented on multi- channel mode identification, the oscillation frequency and damping ratio of the system need to be identified by applying the algorithm to the measurements from each PMU one by one. In theory, since the PMUs are allocated within the system, they should have the same system oscillation mode at a certain operating point such that the identified oscillation frequency from all PMUs should keep consistent. However, in practical applications, the oscillation parameters, especially the damping ratios, identified by measurement data from different PMUs are different due to inevitable measurement errors. To address this issue, [19] used multiple PMU measurement data to improve the identification accuracy of oscillation modes. However, this method decreases the computational efficiency and impedes its applicability in bulk power systems. Therefore, this paper introduces SVD to compress the data from multiple PMUs such that the data size for the CWTbased algorithm can be significantly reduced. Then, the proposed CWT-based algorithm in Section 2 can be applied to identify the dominant oscillation modes of the system.

\subsection{Measurement data compression using SVD}

The procedure of compressing measurement data based on SVD are as follows.

1) Select $r$ channels from $l$ WAMS channels to form a WAMS covariance matrix $\boldsymbol{C} \in \mathbf{R}^{l r \times N}$

$\boldsymbol{C}=\left[\begin{array}{cccc}C_{11}(1) & C_{11}(2) & \cdots & C_{11}(N) \\ C_{12}(1) & C_{12}(2) & \cdots & C_{12}(N) \\ \vdots & \vdots & \ddots & \vdots \\ C_{l r}(1) & C_{l r}(2) & \cdots & C_{l r}(N)\end{array}\right]$

2) Perform SVD on the covariance matrix $\boldsymbol{C}$

$$
\boldsymbol{C}=\boldsymbol{U} \boldsymbol{S} \boldsymbol{V}^{\mathrm{T}}=\left[\begin{array}{ll}
U_{m} & U_{l r-m}
\end{array}\right]\left[\begin{array}{cc}
S_{m} & 0 \\
0 & S_{l r-m}
\end{array}\right]\left[\begin{array}{c}
V_{m}^{\mathrm{T}} \\
V_{l r-m}^{\mathrm{T}}
\end{array}\right]
$$

3) Reconstruct the WAMS covariance matrix based on the results of SVD

$\boldsymbol{C}^{\prime}=\boldsymbol{U}_{m} \boldsymbol{S}_{m} \boldsymbol{V}_{m}^{\mathrm{T}}$

\subsection{Model order determination}

The model order of the system $m$ can be obtained by SVD in (15). However, the submatrix of $\boldsymbol{S}, \boldsymbol{S}_{l r-m}$ is a nonzero matrix due to the impact of measurement noises and errors, leading $S$ to be a full-rank matrix. Thus, in this case, SVD is not applicable to estimate the system model order. To address this challenge, the largest drop in singular values is introduced to identify the system mode. The largest drop based model order determination method has been applied to select the appropriate model order in subspace identification method. In this paper, the largest drop method is further employed to compress the measurement data [16] through the procedure as follows:

1) Calculate the drops of singular values. For the singular value vector $\boldsymbol{S}=\operatorname{diag}\left[\begin{array}{llll}\lambda_{1} & \lambda_{2} & \cdots & \lambda_{l r}\end{array}\right]$, the drop of singular values is calculated as

$\Delta \lambda_{i}=\lambda_{i+1}-\lambda_{i}, i=1,2, \cdots, l r-1$

2) Determine the model order. With the calculated drops of singular, the system model order $m$ can be selected as

$m=\left\{\begin{array}{l}i, \quad \Delta \lambda_{i}=\max (\Delta \lambda), \operatorname{int}\left(\frac{i}{2}\right)=\frac{i}{2} \\ i+1, \quad \Delta \lambda_{i}=\max (\Delta \lambda), \operatorname{int}\left(\frac{i}{2}\right)<\frac{i}{2}\end{array}\right.$

where $\Delta \lambda=\left[\begin{array}{llll}\Delta \lambda_{1} & \Delta \lambda_{2} & \cdots & \Delta \lambda_{l r-1}\end{array}\right]$.

Substituting the estimated $m$ into (15), corresponding $\boldsymbol{U}_{m}, \boldsymbol{S}_{m}$ and $\boldsymbol{V}_{m}$ can be obtained. Then, the reconstructed WAMS covariance matrix $\boldsymbol{C}^{\prime}$ can be obtained by substituting $\boldsymbol{U}_{m}, \boldsymbol{S}_{m}$ and $\boldsymbol{V}_{m}$ into (16).

\subsection{Mode estimation using compressed measurement data}

The coefficient matrix $\boldsymbol{W}_{i j}$ can be obtained by performing CWT on each row vector of $\boldsymbol{C}^{\prime}$ within the range of the frequency band of the power system low-frequency electromechanical oscillation, which is $0.1 \sim 2 \mathrm{~Hz}$.

$$
\boldsymbol{W}_{i}=\left[\begin{array}{cccc}
W_{i}\left(\tau_{1}, t_{1}\right) & W_{i}\left(\tau_{1}, t_{2}\right) & \cdots & W_{i}\left(\tau_{1}, t_{N}\right) \\
W_{i}\left(\tau_{2}, t_{1}\right) & W_{i}\left(\tau_{2}, t_{2}\right) & \cdots & W_{i}\left(\tau_{2}, t_{N}\right) \\
\vdots & \vdots & \ddots & \vdots \\
W_{i}\left(\tau_{p}, t_{1}\right) & W_{i}\left(\tau_{p}, t_{2}\right) & \cdots & W_{i}\left(\tau_{p}, t_{N}\right)
\end{array}\right]
$$


where $p$ is the number of dilation; $N$ is the number of samples; $i$ is the order.

Reordering the $m$ coefficient matrix by dilation, we have

$\boldsymbol{D}_{i k}=\left[\begin{array}{cccc}W_{i 1}\left(\tau_{k}, t_{1}\right) & W_{i 1}\left(\tau_{k}, t_{2}\right) & \cdots & W_{i 1}\left(\tau_{k}, t_{N}\right) \\ W_{i 2}\left(\tau_{k}, t_{1}\right) & W_{i 2}\left(\tau_{k}, t_{2}\right) & \cdots & W_{i 2}\left(\tau_{k}, t_{N}\right) \\ \vdots & \vdots & \ddots & \vdots \\ W_{i m}\left(\tau_{k}, t_{1}\right) & W_{i m}\left(\tau_{k}, t_{2}\right) & \cdots & W_{i m}\left(\tau_{k}, t_{N}\right)\end{array}\right]$

where $k$ is the present dilation.

Performing SVD on $\boldsymbol{D}_{i k}$, we have

$\boldsymbol{D}_{i k}=\boldsymbol{U}_{i k} \boldsymbol{S}_{i k} \boldsymbol{V}_{i k}^{\mathrm{T}}$

where $\boldsymbol{U}_{i k}=\left[\begin{array}{llll}\boldsymbol{U}_{i k 1} & \boldsymbol{U}_{i k 2} & \cdots & \boldsymbol{U}_{i k m}\end{array}\right]$,

$\boldsymbol{V}_{i k}=\left[\begin{array}{llll}\boldsymbol{V}_{i k 1} & \boldsymbol{V}_{i k 2} & \cdots & \boldsymbol{V}_{i k m}\end{array}\right]$,

$\boldsymbol{S}_{i k}=\operatorname{diag}\left[\boldsymbol{S}_{i k s} \quad \mathbf{0}\right]$

$\boldsymbol{S}_{i k s}=\left[\begin{array}{llll}\lambda_{i k 1} & \lambda_{i k 2} & \cdots & \lambda_{i k s}\end{array}\right]$.

Construct vector $\boldsymbol{J}$ based on the first singular value of $\boldsymbol{D}_{\mathrm{ik}}$,

$\boldsymbol{J}=\left[\begin{array}{llll}\lambda_{i 11} & \lambda_{i 21} & \cdots & \lambda_{i p 1}\end{array}\right]$

Calculate oscillation frequency and damping ratio by substituting the right eigenvalue vector $\boldsymbol{V}_{i k j}$, which corresponds to the maximum value in $\boldsymbol{J}$, into (23).

$$
\left\{\begin{array}{l}
\omega_{n i}=\sqrt{\left(\frac{\mathrm{d}\left(\ln \left|\boldsymbol{V}_{i k j}\right|\right)}{\mathrm{d} t}\right)^{2}+\left(\frac{\mathrm{d}\left(\operatorname{angle}\left(\boldsymbol{V}_{\mathrm{ikj}}\right)\right)}{\mathrm{d} t}\right)^{2}} \\
f_{i}=\frac{\omega_{n i}}{2 \pi} \\
\varsigma_{i}=-\frac{\frac{\mathrm{d}}{\mathrm{d} t}\left(\ln \left|\boldsymbol{V}_{i k j}\right|\right)}{\omega_{n i}}
\end{array}\right.
$$

\subsection{Parameter settings in the proposed MCWT approach}

Similar to other mode estimation methods, the performance of MCWT relies on the choice of parameters. The parameters of the proposed MCWT consist of mother wavelet function, center frequency, dilation and translation.

Since all of the above parameters have direct impact on the estimation accuracy, determining appropriate parameter values become critical.

\section{1) Mother wavelet function}

There are several different types of mother wavelet functions in CWT, such as Haar, Meyer, Gaussian, Shannon, Morlet and complex Morlet. The mother wavelet function can be selected according to the characteristics of the analyzed signals. Reference [12] has demonstrated that the complex Morlet wavelet is suitable for mode identification in power system since it is able to reveal these signals in both time and frequency domains Therefore, the complex Morlet wavelet is adopted as the mother wavelet function $\Psi_{\tau, s}$ in the proposed MCWT approach.

\section{2) Center frequency}

The center frequency is another critical parameter that affects estimation accuracy. The center frequency can be approximated as the low-frequency electromechanical oscillation frequencies of typical power systems. In practice, the typical ranges of electromechanical oscillation frequencies are usually considered as known in advance. In general, local electromechanical oscillation modes tend to be within the range from $1 \mathrm{~Hz}$ to $2 \mathrm{~Hz}$ while inter-area ones tend to be within $0.2 \mathrm{~Hz}$ to $1.0 \mathrm{~Hz}$ range. Since this paper focuses on the inter-area dominant mode identification, the center frequency is set as $0.5 \mathrm{~Hz}$.

\section{3) Dilation}

After choosing mother wavelet function and center frequency, it is necessary to choose a range of dilation $\tau$ in (1). The $\tau$ can be determined as

$\tau=\frac{f_{\mathrm{c}}}{f_{\mathrm{a}} \Delta t}$

where $f_{\mathrm{c}}$ is the center frequency; $\Delta t$ is the sampling interval (the sampling interval $\Delta t$ is always $0.01 \mathrm{~s}$ in China); $f_{\mathrm{a}}$ is the modal frequency (for the inter-area modes, the oscillation frequency is between 0.2 and $1 \mathrm{~Hz}$ ). With the given $f_{\mathrm{c}}, f_{\mathrm{a}}$ and $\Delta t$, the dilation $\tau$ in (1) is calculated as 250 via (24).

\section{4) Translation}

To improve the estimation accuracy of the proposed approach, the translation $s$ in (1) is set to be the length of sampling time window.

\subsection{Procedure of proposed MCWT}

In this subsection, the procedure of the proposed MCWT approach for mode estimation is summarized as follows.

1) Gather the measurement data from PMUs.

2) Form WAMS covariance matrix $\boldsymbol{C}$ through (14).

3) Perform SVD on the covariance matrix $\boldsymbol{C}$ with (15).

4) Determine the model order $m$ via Section 3.2.

5) Reconstruct the WAMS covariance matrix $C^{\prime}$ with (16).

6) Obtain the coefficient matrix $\boldsymbol{W}_{i j}$ by performing CWT on each row vector of $\boldsymbol{C}^{\prime}$.

7) Form matrix $\boldsymbol{D}_{i k}$ via (20).

8) Carry out SVD on $\boldsymbol{D}_{i k}$ and construct vector $\boldsymbol{J}$.

9) Determine the wavelet coefficient of the $i^{\text {th }}$ dominant mode $\boldsymbol{V}_{i k j}$ via the maximum value in $\boldsymbol{J}$.

10) Calculate the frequency and damping ratio of dominant mode according to (23). 


\section{Numerical examples}

In this section, the proposed approach is tested and evaluated with the benchmark system of CSG as shown in Fig. 1. It is noted that CSG is one of the largest AC/DC parallel transmission systems in China, which includes Yunnan (YN), Guizhou (GZ), Guangxi (GX), Hainan (HN) and Guangdong (GD) provincial power grids. Electric energy is transmitted from YN, GZ and GX to the load center in GD through an interface consisting of five HVDC lines and three AC corridors [8].

Years of operation experiences and contingency reports indicate that there are two dominant inter-area oscillatory modes, YN-GD and YN-GZ oscillation modes, which are major threats to the system stability of CSG. The former one is at $0.30 \sim 0.43 \mathrm{~Hz}$ with $9.9 \% \sim 18.6 \%$ damping, and the latter one is at $0.48 \sim 0.60 \mathrm{~Hz}$ with $9.5 \% \sim 15.8 \%$ damping $[8,20]$. Several control strategies have been implemented to enhance the stability of CSG including PSS, HVDC coordinated control, etc. Nevertheless, several recent notable oscillation events have brought the real-time monitoring of the inter-area modes to CSG's attention.

\subsection{Simulation data}

According to the operation experiences and contingency reports, the Gao-Zhao HVDC blocking can excite these two inter-area modes. Therefore, the Gao-Zhao HVDC blocking is adopted to evaluate the applicability of the proposed approach. The oscillations of the rotor angles for the critical generators in CSG under this contingency are illustrated in Fig. 2. Since both of the inter-area modes

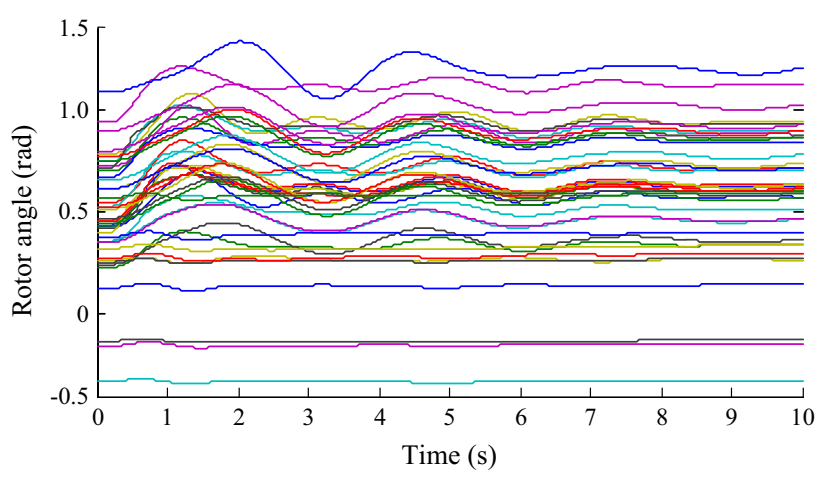

Fig. 2 Oscillation curves of rotor angle

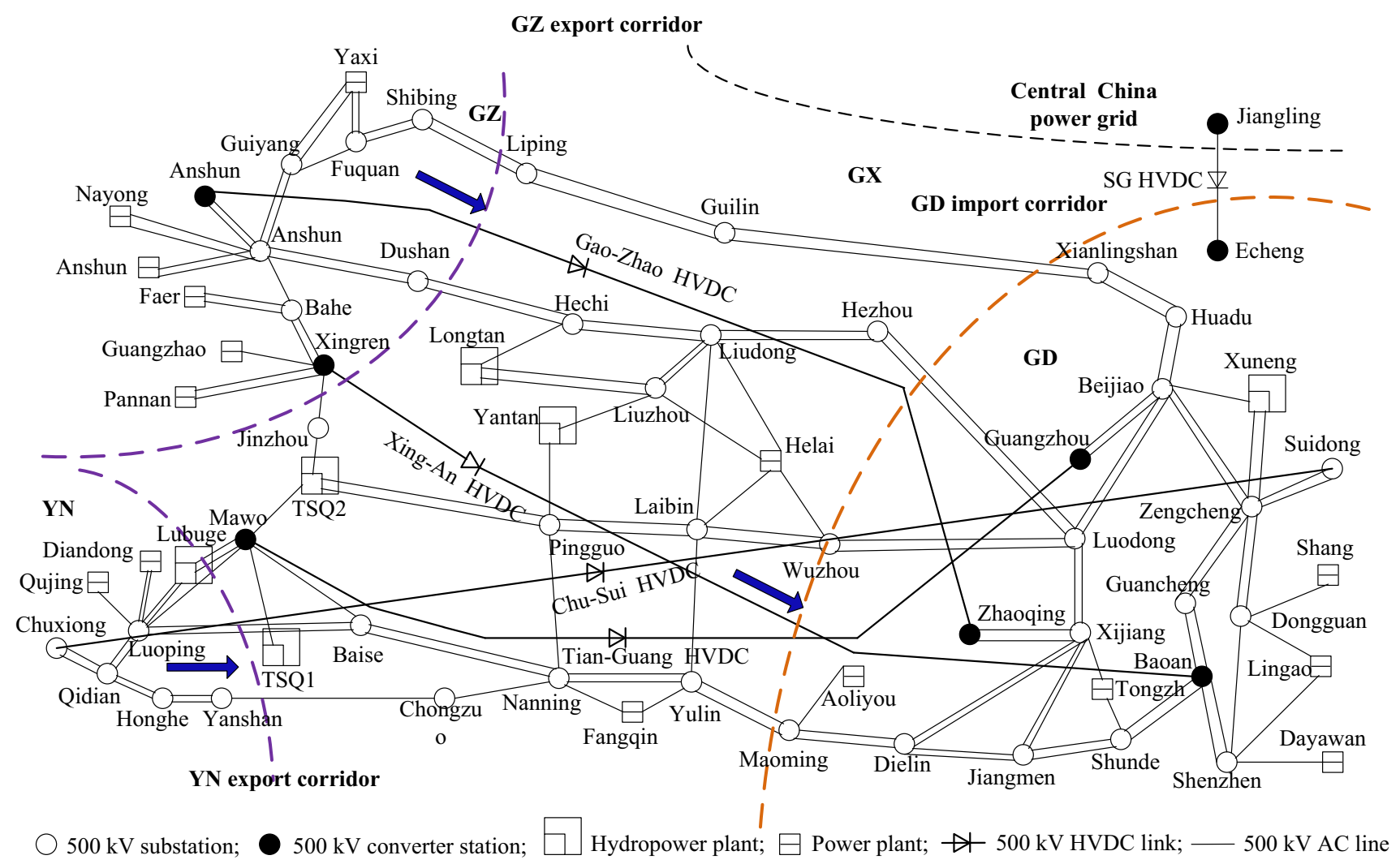

Fig. 1 Schematic diagram of CSG 
Table 1 Three AC interfaces

\begin{tabular}{ll}
\hline $\begin{array}{l}\text { AC } \\
\text { interface }\end{array}$ & Tie-line \\
\hline YN & $\begin{array}{c}\text { LM(Luoping-Mawo), LB(Luoping-Baise), } \\
\text { YC(Yanshan-Chongzuo) }\end{array}$ \\
GZ & $\begin{array}{c}\text { SL(Shibing-Liping), SH(Dushan-Hechi), TJ(TSQ2- } \\
\text { Jinzhou) }\end{array}$ \\
GD & WL(Wuzhou-Luodong), HL(Hezhou-Luodong), \\
& YM(Yulin-Maoming), MD(Maoming-Dielin), \\
& GS(Guilin-XianlingShan)
\end{tabular}

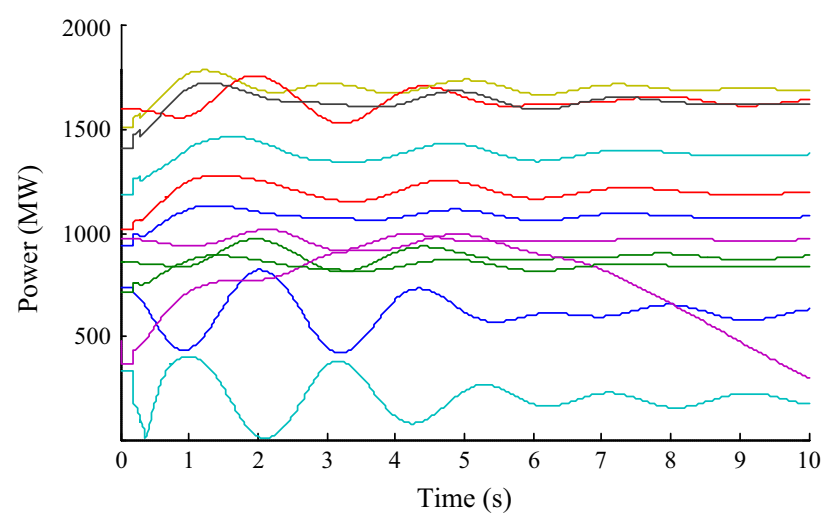

Fig. 3 Oscillation curves of active power on tie-lines

have high observability on the three AC interfaces [8, 20], the active power data of tie-lines of AC interfaces listed in Table 1 are selected as the input data of the proposed approach. The oscillation curves of the active power for the selected tie-lines are demonstrated in Fig. 3.
Following the proposed procedure in Section 3.5, the auto-covariance for the active power of tie-line in Fig. 3 can be calculated. Then, the proposed MCWT is adopted to identify the dominant modes from the auto-covariance. The results are shown in Table 2. Also, these results are compared with those obtained by N4SID and Prony to demonstrate the effectiveness of the proposed method. It can be observed that the oscillation frequency and damping ratio are feasible and accurate. The YN-GD and YN-GZ modes excited by Gao-Zhao HVDC blocking can be identified by the tie-lines in Table 1 .

From Table 2, it can be observed that all the methods can identify the oscillation frequency and damping ratio of CSG from the tie-lines in Table 1. However, there are differences among the identification results, especially for the damping ratios of the oscillation mode. As shown in Table 2, the oscillation frequencies identified by MCWT are basically consistent but the damping ratios are different. For instance, the damping ratio of the YN-GZ mode identified from tie-line YM is roughly half of the damping ratios identified from other tie-lines. Similar phenomenon can be found in the results obtained by N4SID and Prony. This fact prevents system operators from monitoring system dynamic stability accurately.

This issue can be solved by the proposed method with the following procedure. The active power on the tie-lines of the interface is taken as input for the covariance matrix in (14). Then, the covariance matrix is obtained with all involved channels. According to the number of tie-lines of each interface in Table 1, the dimensions of the covariance matrix are 9, 9 and 25 for $\mathrm{YN}, \mathrm{GZ}$, and GD interfaces respectively. The eigenvalues obtained by performing SVD on the three covariance matrix are shown in Fig. 4. Further,

Table 2 Identification results from different methods under Gao-Zhao HVDC blocking

\begin{tabular}{|c|c|c|c|c|c|c|c|c|c|c|c|c|}
\hline \multirow[t]{2}{*}{ Tie-line } & \multicolumn{2}{|c|}{$\begin{array}{l}\text { CWT (YN-GD } \\
\text { mode) }\end{array}$} & \multicolumn{2}{|c|}{$\begin{array}{l}\text { CWT (YN-GZ } \\
\text { mode) }\end{array}$} & \multicolumn{2}{|c|}{$\begin{array}{l}\text { N4SID (YN-GD } \\
\text { mode) }\end{array}$} & \multicolumn{2}{|c|}{$\begin{array}{l}\text { N4SID (YN-GZ } \\
\text { mode) }\end{array}$} & \multicolumn{2}{|c|}{$\begin{array}{l}\text { Prony (YN-GD } \\
\text { mode) }\end{array}$} & \multicolumn{2}{|c|}{$\begin{array}{l}\text { Prony (YN-GZ } \\
\text { mode) }\end{array}$} \\
\hline & $f(\mathrm{~Hz})$ & Damping & $f(\mathrm{~Hz})$ & Damping & $f(\mathrm{~Hz})$ & Damping & $f(\mathrm{~Hz})$ & Damping & $f(\mathrm{~Hz})$ & Damping & $f(\mathrm{~Hz})$ & Damping \\
\hline $\mathrm{YC}$ & 0.3584 & 0.1075 & 0.4763 & 0.0795 & 0.3442 & 0.1300 & 0.4852 & 0.0848 & 0.3510 & 0.1528 & 0.4620 & 0.1034 \\
\hline LM & 0.3616 & 0.1067 & 0.4821 & 0.0840 & 0.3380 & 0.1252 & 0.5050 & 0.0848 & 0.3460 & 0.1291 & 0.4970 & 0.0756 \\
\hline LB & 0.3507 & 0.1051 & 0.4642 & 0.0774 & 0.3409 & 0.1287 & 0.4847 & 0.0401 & 0.3380 & 0.1497 & 0.4800 & 0.0229 \\
\hline $\mathrm{TJ}$ & 0.3385 & 0.1105 & 0.4763 & 0.0795 & 0.3365 & 0.1412 & 0.5027 & 0.0783 & 0.3430 & 0.1277 & 0.4940 & 0.1049 \\
\hline SH & 0.3412 & 0.1011 & 0.4968 & 0.0786 & 0.3389 & 0.0679 & 0.4955 & 0.0728 & 0.3460 & 0.1172 & 0.4950 & 0.0934 \\
\hline SL & 0.3247 & 0.1123 & 0.5018 & 0.0950 & 0.3477 & 0.1124 & 0.5257 & 0.0794 & 0.3490 & 0.1325 & 0.5430 & 0.1088 \\
\hline GS & 0.3344 & 0.1076 & 0.5044 & 0.0911 & 0.3411 & 0.1224 & 0.5313 & 0.1010 & 0.3470 & 0.1402 & 0.5460 & 0.0961 \\
\hline YM & 0.3388 & 0.1100 & 0.4932 & 0.0572 & 0.3307 & 0.1400 & 0.4956 & 0.0532 & 0.3480 & 0.1319 & 0.5060 & 0.0438 \\
\hline MD & 0.3353 & 0.1027 & 0.5041 & 0.0962 & 0.3404 & 0.1168 & 0.5265 & 0.0920 & 0.3480 & 0.1365 & 0.5330 & 0.1346 \\
\hline WL & 0.3346 & 0.1065 & 0.4967 & 0.0919 & 0.3411 & 0.1128 & 0.4975 & 0.0849 & 0.3480 & 0.1252 & 0.4970 & 0.0790 \\
\hline HL & 0.3485 & 0.1097 & 0.5087 & 0.0907 & 0.3442 & 0.1300 & 0.4852 & 0.0848 & 0.3510 & 0.1528 & 0.4620 & 0.1034 \\
\hline
\end{tabular}




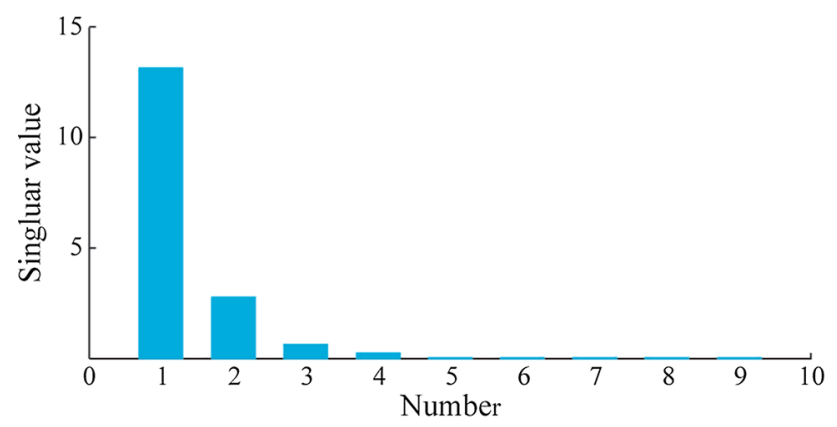

(a) $\mathrm{YN}$

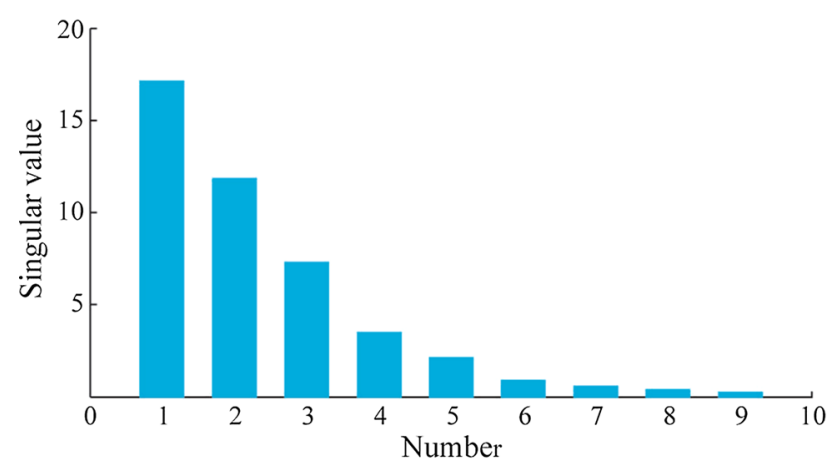

(b) GZ

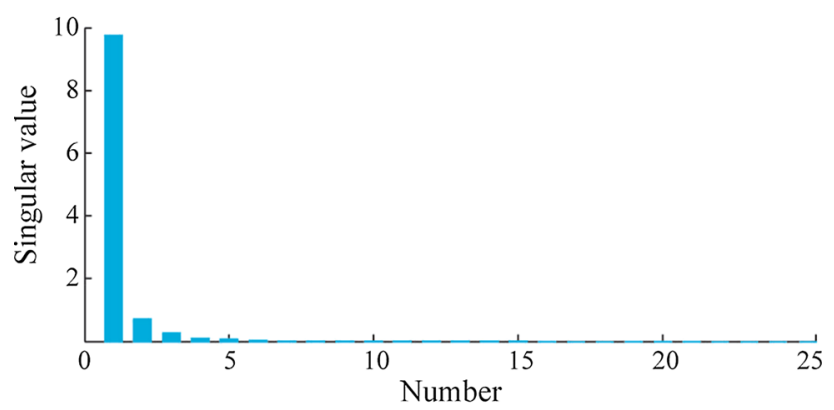

(c) GD

Fig. 4 Singular values of SVD

Fig. 5 shows the drops of eigenvectors. According to (18), the system model order is set to 2 based on the largest drop method. Based on the determined system model order, the reduced-order covariance matrix can be obtained according to (16), and the compressed measurement data are shown in Fig. 6.

CWT is performed on the signals in Fig. 6 to form the coefficient matrix $\boldsymbol{D}_{i k}$ in (20). Further, SVD is applied to $\boldsymbol{D}_{i k}$ and the right singular value that corresponds to the maximum singular value is retained. Finally, the singularvalue vector $\boldsymbol{J}$ can be obtained consisting of all maximum singular value as shown in Fig. 7. According to Section 3.3, the right eigenvector $\boldsymbol{V}_{i k j}$ corresponding to the maximum eigenvalue in $\boldsymbol{J}$ is taken as an input for (23). The results of identifying the right eigenvector $\boldsymbol{V}_{i k j}$ based on CWT are shown in Table 3. From Table 3, the identified

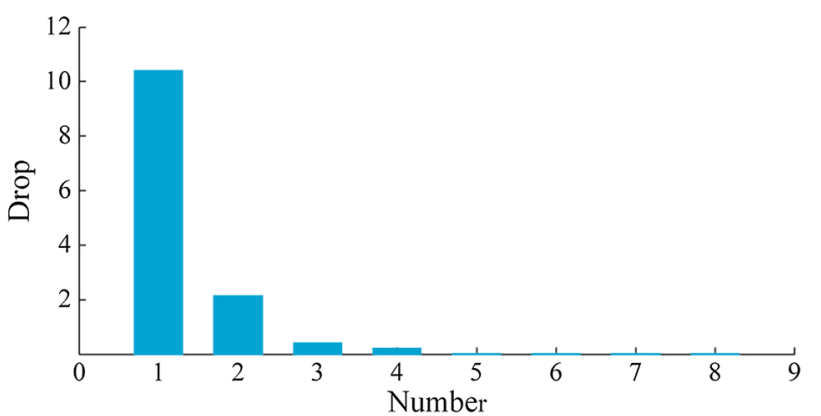

(a) YN

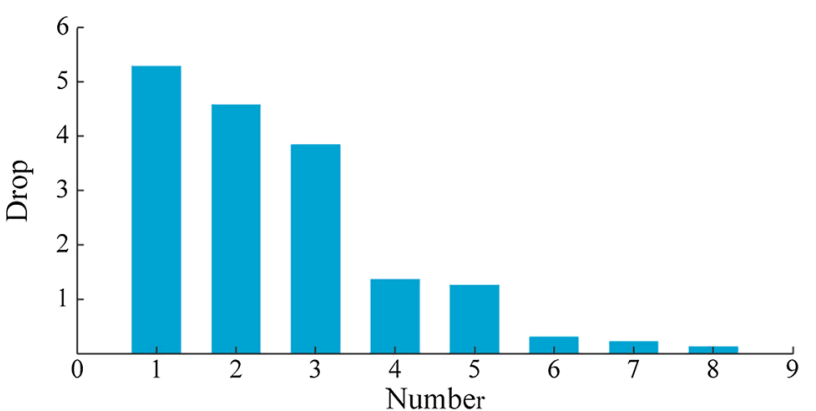

(b) GZ

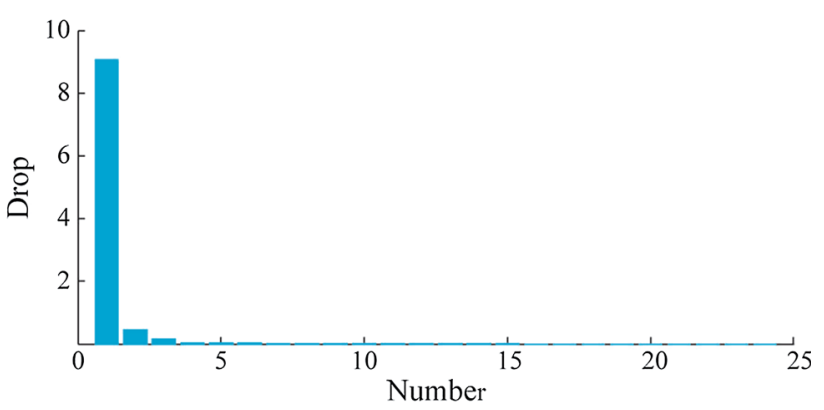

(c) GD

Fig. 5 Drops of Singular values

oscillation frequencies and damping ratios from the oscillations on YN-GD and YN-GZ excited by Gao-Zhao HVDC blocking are $0.3584 \mathrm{~Hz}$ with 0.1088 damping, $0.3473 \mathrm{~Hz}$ with 0.1090 damping, $0.3408 \mathrm{~Hz}$ with 0.1100 , $0.4886 \mathrm{~Hz}$ with 0.0847 damping, $0.4928 \mathrm{~Hz}$ with 0.0897 damping, and $0.4935 \mathrm{~Hz}$ with 0.0844 damping. Comparing the results in Table 3 with those in Table 2, it can be concluded that the proposed method is able to identify the oscillation and damping ratios on each interface of the oscillation modes.

Similarly, taking the measurement data from all the tielines in Table 1 as inputs, the identification results can be obtained as $0.3597 \mathrm{~Hz}$ with 0.1096 damping and $0.4664 \mathrm{~Hz}$ with 0.0855 damping. To verify the accuracy of the proposed MCWT, Table 4 further lists the results estimated by the small signal stability analysis (SSSA). Comparing the 


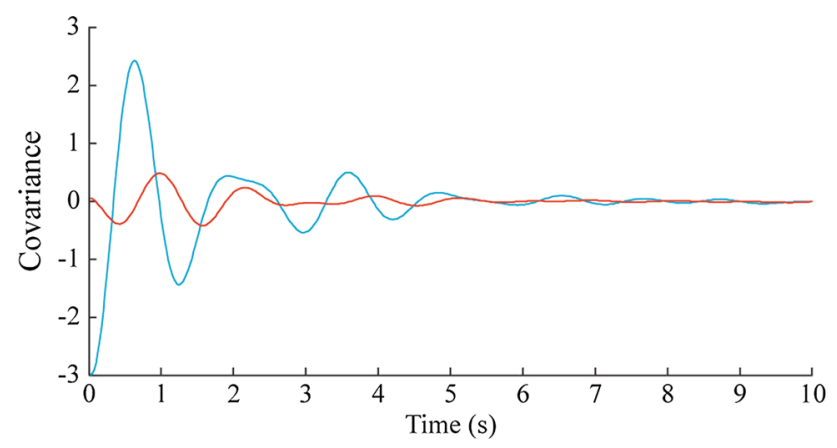

(a) $\mathrm{YN}$

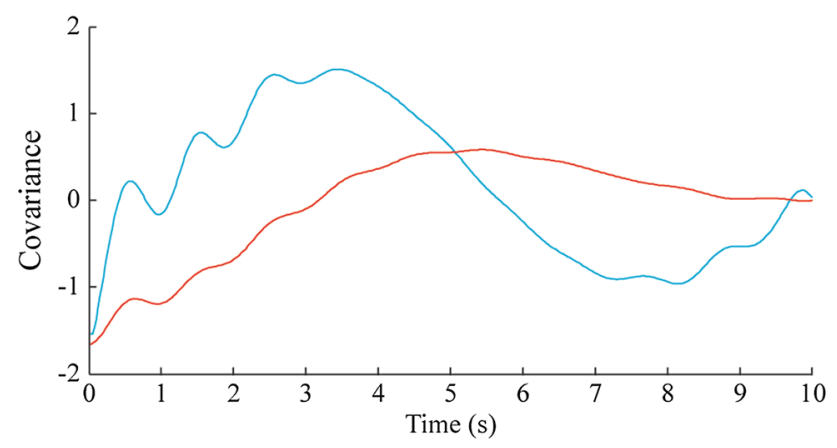

(b) GZ

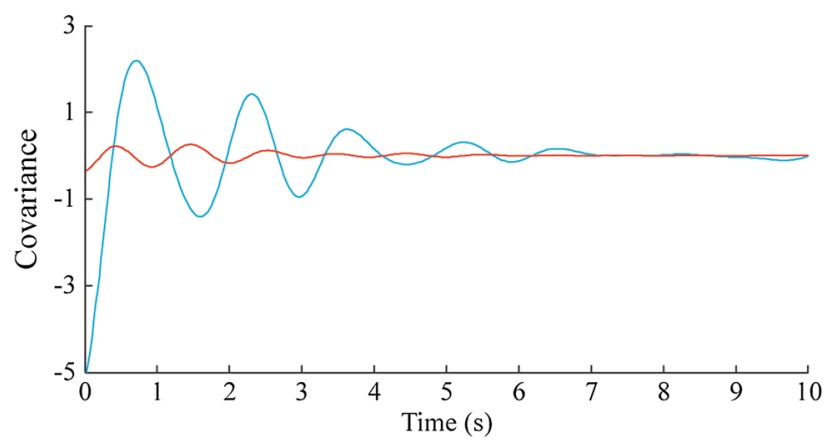

(c) GD

- Auto-covariance; Cross-covariance

Fig. 6 Compressed and constructed signals

estimation results obtained by SSSA and the proposed method in Table 4, it can be concluded that the proposed approach can accurately identify the dominant modes. Moreover, since the damping ratios are all larger than 0.05 , the oscillations among regions in CSG caused by Gao-Zhao HVDC blocking is considered to be stable.

It is noted that, from the view of methodology, the conventional CWT, N4SID, and Prony are based on single-channel data. Data compression is not involved in those methods such that the impact of measurement noises and calculation errors cannot be mitigated. In addition, using the measurement data from a single channel only reflects the local dynamic features of the

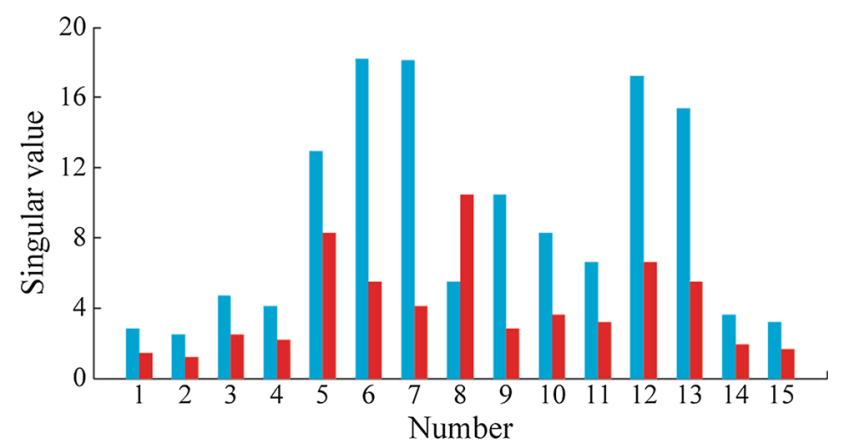

(a) $\mathrm{YN}$

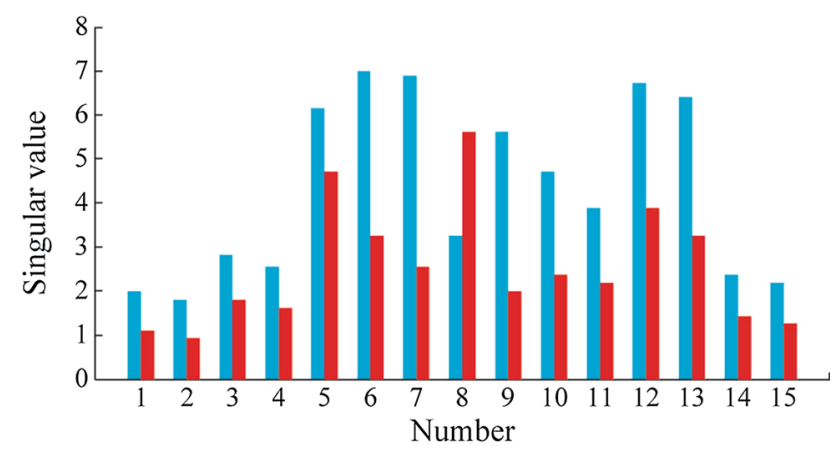

(b) GZ

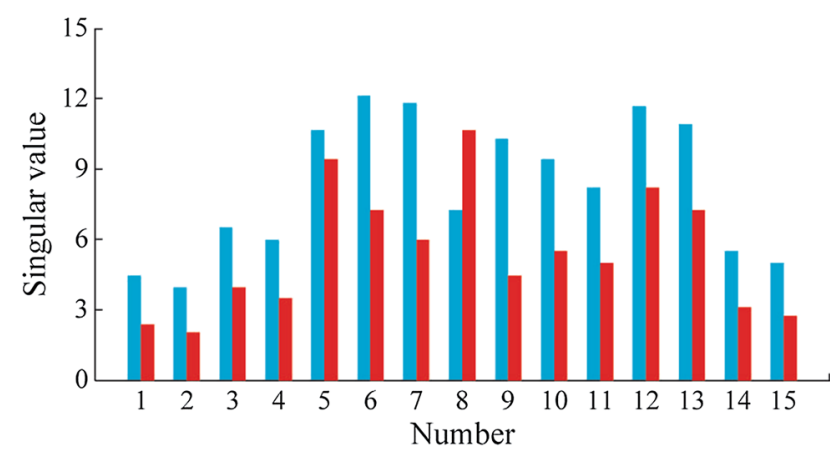

(c) GD

Auto-covariance; Cross-covariance

Fig. 7 Maximum singular value vectors

Table 3 Identified oscillation mode using proposed MCWT approach

\begin{tabular}{llllll}
\hline Interface & \multicolumn{2}{l}{ YN-GD mode } & & \multicolumn{2}{l}{ YN-GZ mode } \\
\cline { 2 - 3 } & $f(\mathrm{~Hz})$ & Damping & & $f(\mathrm{~Hz})$ & Damping \\
\hline YN & 0.3584 & 0.1088 & 0.4886 & 0.0847 \\
GZ & 0.3473 & 0.1090 & & 0.4928 & 0.0897 \\
GD & 0.3408 & 0.1100 & & 0.4935 & 0.0844 \\
\hline
\end{tabular}

system, leading to less accurate results. The proposed CWT method is based on multi-channel measurement data such that it can capture the global dynamic features, 
reflecting the real system dynamics more accurately. In addition, through the data compression and reconstruction, the proposed method is effective in mitigating the impact of noises and errors. Therefore, the proposed method is able to generate better results than the other three.

\subsection{Field-measurement data}

In the previous subsection, the proposed method is demonstrated to be effective in identifying the oscillation frequencies and damping ratios. This subsection will demonstrate the applicability of the proposed method in bulk power systems using the field-measurement data from CSG. Figure 8 shows the recorded active power data of PMUs at LP, LD, CZ, SZ, JZ and SD in the contingency at 16:07:10.007 on August 11, 2012.

Firstly, the covariance of the active power on each branch is calculated as the input to the CWT algorithm. Then, the identified oscillation frequency and damping are compared with the results obtained by N4SID and Prony, as shown in Fig. 9. It can be revealed from Fig. 9 that this contingency excited YN-GD oscillation mode of CSG. In addition, the results indicate that the identification results of the proposed method are the same with those of N4SID and Prony.

Further, the active power data collected by each PMU in substations is the input to the covariance matrix in (19), and the data are compressed according to (18). Then, SVD is implemented to the coefficient matrix, and CWT is performed on the compressed measurement data to form the coefficient matrix. The identification results in Table 5 can be obtained following the procedure described in Subsection 3.3. It is evident in Table 5 that this contingency only excites the YN-GD inter-area oscillation mode, whose frequency and damping are around $0.35 \mathrm{~Hz}$ and 0.07 respectively, captured by LP, LD, CZ, SZ, JZ and SD. All the active power in Fig. 8 is considered as the input of the proposed MCWT approach, and the estimated frequency and damping ratio are $0.3580 \mathrm{~Hz}$ and 0.0689 of the YN-GD mode. According to the estimation results, it is clear that CSG is stable in term of YN-GD mode.

Table 4 Comparison with SSSA

\begin{tabular}{llllll}
\hline Method & \multicolumn{2}{l}{ YN-GD mode } & & \multicolumn{2}{l}{ YN-GZ mode } \\
\cline { 2 - 3 } \cline { 5 - 6 } & $f(\mathrm{~Hz})$ & Damping & & $f(\mathrm{~Hz})$ & Damping \\
\hline MCWT & 0.3597 & 0.1096 & & 0.4664 & 0.0855 \\
SSSA & 0.3624 & 0.1058 & & 0.4801 & 0.0913 \\
\hline
\end{tabular}

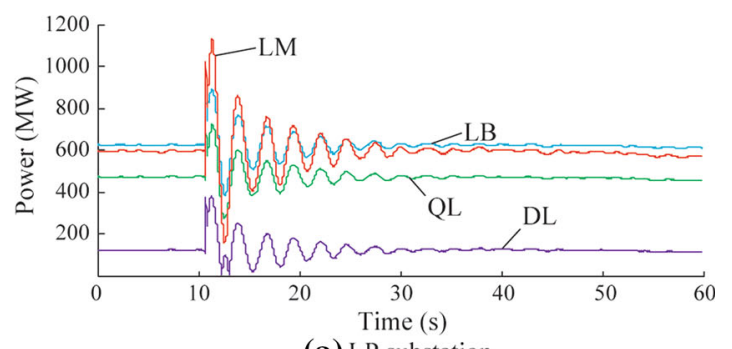

(a) LP substation

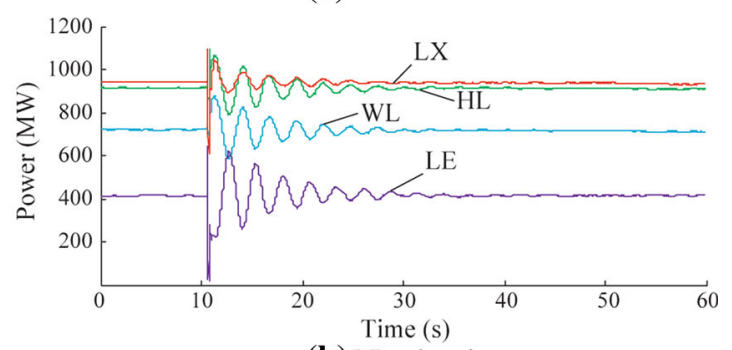

(b) LD substation

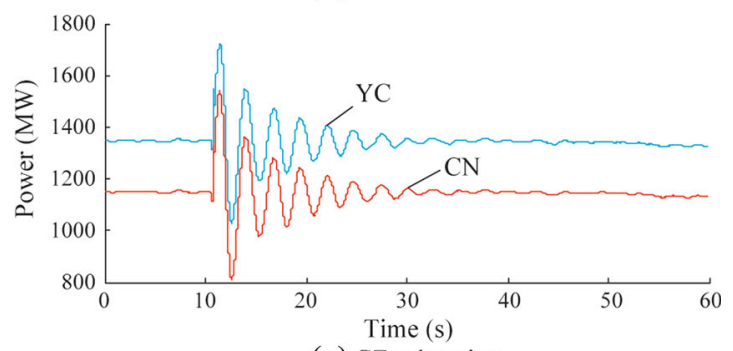

(c) CZ substation

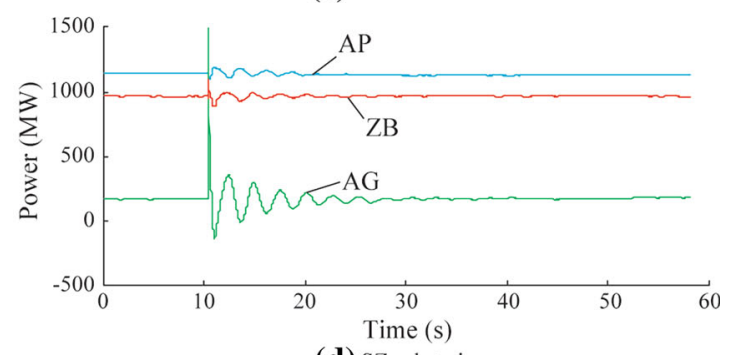

(d) SZ substation

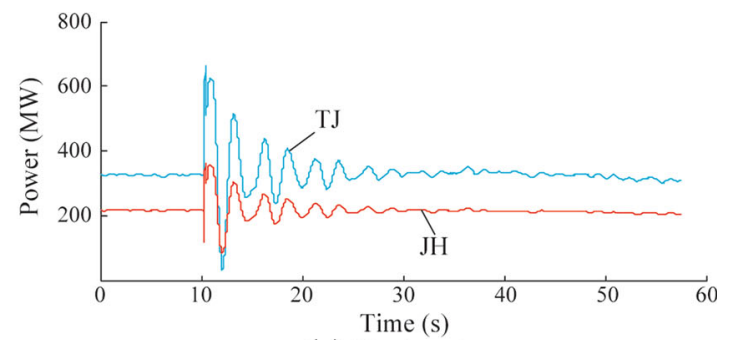

(e) JZ substation

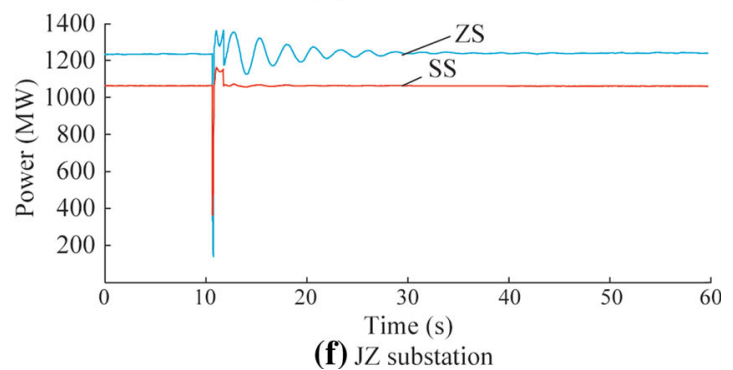

Fig. 8 Recorded data of WAMS under a branch contingency 


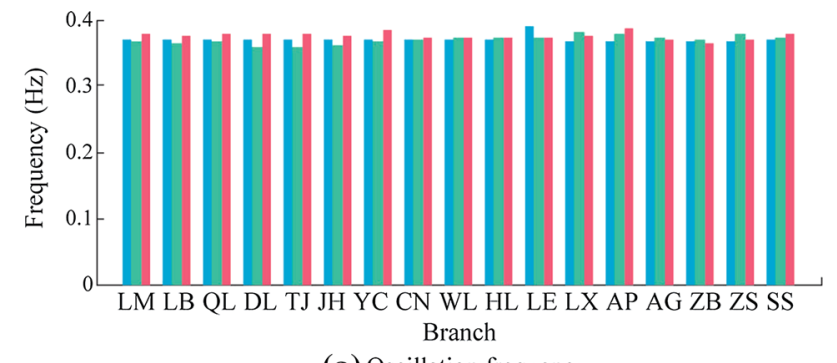

(a) Oscillation frequency

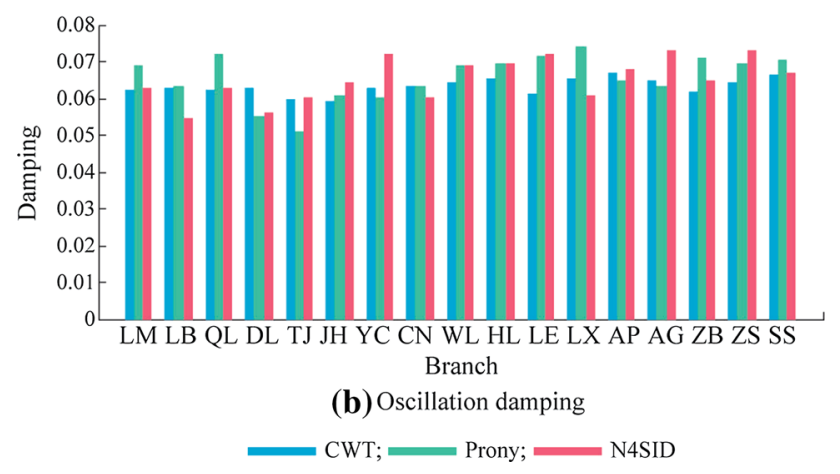

Fig. 9 Comparison of results by CWT, Prony and N4SID

Table 5 Identified oscillation mode using MCWT

\begin{tabular}{lll}
\hline Substation & \multicolumn{2}{l}{ YN-GD mode } \\
\cline { 2 - 3 } & $f(\mathrm{~Hz})$ & Damping \\
\hline LP & 0.3776 & 0.0638 \\
LD & 0.3776 & 0.0658 \\
CZ & 0.3772 & 0.0646 \\
SZ & 0.3780 & 0.0676 \\
JZ & 0.3776 & 0.0690 \\
SD & 0.3746 & 0.0679 \\
\hline
\end{tabular}

\section{Conclusion}

A multi-channel continuous wavelet transform approach to estimating dominant oscillation mode is developed in this paper to holistically assess power system dynamic stability. The proposed approach is evaluated with both simulation data and field-measurement data from CSG to verify its accuracy and effectiveness. The test results demonstrate that the proposed approach outperforms the conventional CWT, and it is applicable to the mode estimation on multi-channel filed-measurement data with less computational burden. Therefore, the proposed MCWT mode estimation approach is capable of holistically estimating dominant oscillation modes to capture the dynamic features of bulk power systems.
Open Access This article is distributed under the terms of the Creative Commons Attribution 4.0 International License (http:// creativecommons.org/licenses/by/4.0/), which permits unrestricted use, distribution, and reproduction in any medium, provided you give appropriate credit to the original author(s) and the source, provide a link to the Creative Commons license, and indicate if changes were made.

\section{References}

[1] Kundur P, Paserba J, Ajjarapu V et al (2004) Definition and classification of power system stability IEEE/CIGRE joint task force on stability terms and definitions. IEEE Trans Power Syst 19(3):1387-1401

[2] Task Force on Identification of Electromechanical Modes (2012) Identification of electromechanical modes in power systems. PES-TR15. IEEE PES Resource Center, Piscataway, NJ, USA

[3] Jiang T, Yuan HY, Jia HJ et al (2015) Stochastic subspace identification-based approach for tracking inter-area oscillatory modes in bulk power system utilizing synchrophasor measurements. IET Gener Transm Distrib 9(15):2409-2418

[4] Zhou N, Pierre JW, Hauer JF (2006) Initial results in power system identification from injected probing signals using a subspace method. IEEE Trans Power Syst 21(3):1296-1302

[5] Kundur P (1994) Power system stability and control. Mc-GrawHill, New York, NY, USA

[6] Venkatasubramanian V, Schattler H, Zaborsky J (1995) Dynamic of large constrained nonlinear systems - a taxonomy theory. P IEEE 83(11):1530-1561

[7] Jia HJ, Yu XD, Yu YX et al (2008) Power system small signal stability region with time delay. Int J Electr Power Energ Syst 30(1):16-22

[8] Jiang T, Jia HJ, Zhao JL et al (2014) Mode matching pursuit for estimating dominant modes in bulk power grid. IET Gener Transm Distrib 8(10):1677-1686

[9] Zhou N, Trudnowski DJ, Pierre JW et al (2008) Electromechanical mode online estimation using regularized robust RLS methods. IEEE Trans Power Syst 23(4):1670-1680

[10] Zhou N, Pierre JW, Trudnowski D (2012) A stepwise regression method for estimating dominant electromechanical modes. IEEE Trans Power Syst 27(2):1051-1059

[11] Hauer JF, Demeure CJ, Scharf LL (1990) Initial results in Prony analysis of power system response signals. IEEE Trans Power Syst 5(1):80-89

[12] Rueda JL, Juárez CA, Erlich I (2011) Wavelet-based analysis of power system low-frequency electromechanical oscillations. IEEE Trans Power Syst 26(3):1733-1743

[13] Ni JM, Shen C, Liu F (2011) Estimating the electromechanical oscillation characteristics of power system based on measured ambient data utilizing stochastic subspace method. In: Proceedings of the 2009 IEEE power and energy society general meeting, San Diego, CA, USA, 24-29 Jul 2011, 7p

[14] Turunen J (2011) A wavelet-based method for estimating damping in power systems. Ph D Thesis. Aalto University, Espoo, Finland

[15] Khalilinia H, Vaithianathan V (2015) Modal analysis of ambient PMU measurements using orthogonal wavelet bases. IEEE Trans Smart Grid 6(6):2954-2963

[16] Khairudin Qudaih Y, Watanabe M et al (2015) Synchrophasor measurement based damping estimation and oscillation mode detection using FFT-CWT approach in Japan campus WAMS. Int J Smart Grid Clean Energ 4(2):93-102 
[17] Xiao J, Bai LQ, Li FX et al (2014) Sizing of energy storage and diesel generators in an isolated microgrid using discrete Fourier transform (DFT). IEEE Trans Sustain Energ 5(3):907-916

[18] Xiao J, Bai LQ, Wang CS et al (2012) Method and software for planning and designing of microgrid. P CSEE 32(25):149-157

[19] Zhang JH, Nabavi S, Chakrabortty A, et al (2015) Convergence analysis of ADMM based power system mode estimation under asynchronous wide-area communication delays. In: Proceedings of the IEEE PES general meeting, Denver, CO, USA, 26-30 Jul 2015, 5 pp

[20] CSG Dispatching and Control Center (2013) Operation mode of CSG in 2013'. China Southern Power Grid, Guangzhou, China

Tao JIANG received the B.S. and M.S. degrees in electrical engineering from Northeast Dianli University, Jilin, China, in 2006 and 2011, respectively, and the Ph.D degree in electrical engineering from Tianjin University, Tianjin, China, in 2015. He is presently an Associate Professor with the Department of Electrical Engineering, Northeast Dianli University, Jilin, JL, China. He was with the Department of Electrical and Computer Engineering, North Carolina State University, Raleigh, NC, USA, as a visiting scholar from 2014 to 2015. His research interests include power system stability analysis and control, renewable energy integration, demand response, and smart grid.

Linquan BAI is a Ph.D student at The University of Tennessee, Knoxville. He received his B.S. and M.S degrees from Tianjin University in 2010 and 2013 respectively. His research interests include power market, voltage stability, energy storage applications, and microgrid energy management.

Guoqing LI received the $\mathrm{Ph} . \mathrm{D}$ degrees in electrical engineering from Tianjin University, Tianjin, China, in 1998. He is a professor of Northeast Dianli University. His research interests include power system stability analysis and control, distribution automation, renewable energy integration and smart grids.

Hongjie JIA received the B.S., M.S., and Ph.D degrees in electrical engineering from Tianjin University, Tianjin, China, in 1996, 1998, and 2001, respectively. He is a Professor with Tianjin University. His research interests include power system stability analysis and control, distribution network planning, renewable energy integration, and smart grid.

Qinran HU received the B.S. degree from Southeast University, Nanjing, China, in 2010, and the M.S. and Ph.D degrees from the Department of EECS, The University of Tennessee, Knoxville, TN, USA, in 2013 and 2015, respectively. He is currently a postdoc in Harvard University, Cambridge, MA, USA. His research interests include mechanism design, human behavior analysis, game theory and electricity market.

Haoyu YUAN received the B.S. degree in electrical engineering from Southeast University, Nanjing, China, in 2011. He started his Ph.D study at The University of Tennessee, Knoxville in August 2011. His interests include power system stability and power system economy. 